sympathectomy of the carotid artery in neck region, and the procedure for the dilatation of the diameter of internal carotid artery, sweeping away of some focuses of inflammation. Thereafter some vasodilators and steroids were prescribed.

Though the result of this treatment is not clear yet, but a case of 4 cases treated by this method showed some improvement on the carotid angiogram after one year and three months.

\title{
D-15. On the Net-like Vascular Anomaly of the Cerebral Artery
}

\author{
Nobuo Moriyasu, Shū Nishio, Kohten Satoh and Torao FuJII \\ The Department of Neurosurgery, Nihon University \\ School of Medicine \\ Keiichiro AKaI and Shigeo Hashimoto \\ The Second Department of Pathology, Nihon University \\ School of Medicine
}

Twelve cases of basal net-like vascular anomaly have experienced by us during these eight years. Seven of them are adult cases and another 5 are infants.

In 6 of 7 adult cases, Initial Signs were loss of consciousness of sudden onset due to subarachnoidal hemorrhage. But in a 33-year-old woman, with posttraumatic complaint after traffic accident was demonstrated by angiography the ret-like arterial anomaly by chance.

In infants, the chief complaint was almost often transient hemiplegia and the signs often repeatedly observed in various grade.

The Angiographical finding:-The anterior and middle cerebral arteries bilaterally opacified as incomplete shape and distribution with the net-like vascular finding at the base of cerebrum.

The retrograde filling from the posterior cerebral artery via $\mathrm{Rr}$. Splenii was remarkably recognized.

The connection of contrast medium could not visualized between the rostral trunk of the anterior cerebral artery and the peripheral part of the pericallosal artery opacified by retrograde filling.

In two of infant cases, net-like finding also revealed in the orbital region added to the base of cerebrum.

The Postmortem Examination was performed to a 55 year-old woman with net-like vascular finding. Anterior cerebral arteries and middle cerebral arteries 
and basilar part of posterior communicating arteries were extremely fine and narrow on both sides.

Thrombosis was never recognized in any part. Of the anterior cerebral arteries, pericallosal arteries were also revealed fine cords from the origin on the left and from the frontopolar artery on the right to the central part of the Callosum. Microscopic findings of the wall of fine cord-like arteries demonstrated thickening of intima, thickening and rupture of internal elastic lamella, defect of muscle layer of tunica media. These findings, in other words, were the evident Hypoplasia of the arteries. The arteries of the circle of Willis consisted of the normal and anomalous vessels shown fragmental arrangement in each other. The chief components of the net-like anomaly were anterior choroidal arteries and their large branchings.

We thought, that the pathogenesis of this case is Hypoplasia of arteries, especially the component of Circle of Willis, belonged to the congenital anomaly. The embryonal Preparation could be necessary for such an anomaly. The arterial net-like structure might have assisted the function of the incomplete circle of Willis and cerebral circulation.

This may be the reason that the neurological findings were normal in most of adult cases.

The initial symptom in adult cases usually was subarachnoidal hemorrhage. We have conjectured about the reason that the hemorrhage occurred because of the weakness of arterial wall as a result of secondary changes on the base of the primary vascular anomaly. On the other hand, in infants, the process of secondary changes in arterial wall might not yet advanced, and transient cerebral hypoxia could occurred, then, hemiplegia repeatedly recognized. It could be thought that if patients had no chance to be taken such a hypoxia, they would lived in good health till secondary change of artery occurred. And thus, we think that Pathogenesis of this disease in infant may have similarity with that of alult.

\title{
D-16. On the 'Cerebral Juxta-basal Telangiectasia'
}

\author{
Keiji Sano, Takeko Kuwabara, Minoru Jimbo and Isamu Saito \\ Department of Neurosurgery, Faculty of Medicine, \\ University of Tokyo
}

A peculiar form of cerebral vascular disease which may be called as cerebral juxta-basal telangiectasia and in which some recurrent neurological symptoms and signs such as hemiplegia, aphasia or convulsion is discussed. In the carotid angiogram, obstruction or narrowing of the distal part of the carotid siphon and abnormal 